\title{
Enjeux, contraintes et réalités de l'accueil des personnes en situation de handicap dans les espaces d'éducation relative à l'environnement
}

Cindy Lebat

\section{(2) OpenEdition}

\section{Journals}

Édition électronique

URL : https://journals.openedition.org/ere/3470

DOI : $10.4000 /$ ere.3470

ISSN : 2561-2271

Éditeur

Centr'ERE

Référence électronique

Cindy Lebat, «Enjeux, contraintes et réalités de l'accueil des personnes en situation de handicap dans les espaces d'éducation relative à l'environnement », Éducation relative à l'environnement [En ligne], Volume 15 - 1 | 2019, mis en ligne le 20 décembre 2019, consulté le 15 octobre 2021. URL : http:// journals.openedition.org/ere/3470; DOI : https://doi.org/10.4000/ere.3470

Ce document a été généré automatiquement le 15 octobre 2021

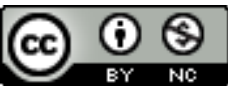

La revue Éducation relative à l'environnement est mise à disposition selon les termes de la Licence Creative Commons Attribution - Pas d'Utilisation Commerciale 4.0 International. 


\title{
Enjeux, contraintes et réalités de l'accueil des personnes en situation de handicap dans les espaces d'éducation relative à l'environnement
}

\author{
Cindy Lebat
}

1 Cet article apporte un éclairage sur la façon dont se croisent deux défis sociétaux majeurs : l'éducation relative à l'environnement d'une part, et la prise en compte des personnes en situation de handicap d'autre part. Il s'agit de se pencher sur la manière dont sont pensés et conçus les activités et les lieux d'éducation relative à l'environnement, notamment dans leur façon d'aborder et de s'emparer de ces deux préoccupations sociétales.

2 Nous appelons lieux d'éducation relative à l'environnement ou espaces naturels formalisés tout espace naturel ou muséographique de mise en contact avec la nature et les problématiques environnementales, développant un objectif de sensibilisation et d'éducation à ces questions. Les problématiques environnementales sont pensées dans une acception large et prennent en considération l'ensemble des liens tissés entre les humains et leur environnement vécu, dans leurs dimensions écologiques, sociales, culturelles. Les espaces d'éducation relative à l'environnement (ERE) peuvent ainsi prendre la forme de lieux aussi variés que des aires naturelles protégées (parcs naturels régionaux ou nationaux, géoparcs, réserves naturelles, etc.), parcs zoologiques, centres d'interprétation, espaces muséaux, muséums d'histoire naturelle, écomusées, fermes pédagogiques, etc. Au sein de chaque espace, les lieux et leurs propositions peuvent se décliner sous des formes diverses. 


\section{La prise en compte des personnes en situation de handicap : une problématique sociétale majeure}

3 Ces dernières années ont vu se développer une prise de conscience considérable concernant le handicap, se traduisant par des mutations conceptuelles et sociales soustendues par un cadre juridique et politique solide. En lien avec ces changements, se développent des enjeux propres à la prise en compte du handicap et au développement de l'accessibilité, que nous allons expliciter. Mais avant cela, commençons par quelques éléments de contextualisation, afin de bien cerner la place qu'occupent les problématiques d'accessibilité dans le débat social contemporain.

4 Tout d'abord et comme le rappelle le Rapport Mondial sur le Handicap, notons que «le handicap fait partie de la condition humaine " (Banque Mondiale et OMS, 2011, p. 3) : non seulement il concerne une grande partie de la population, mais il fait référence à une diversité de réalités (de la simple gêne à l'invalidité complète) qui l'ancre dans des sphères sociales multiples. S'il est souvent associé au seul handicap moteur (le pictogramme du fauteuil roulant étant quasiment toujours utilisé pour désigner l'ensemble des personnes en situation de handicap, sur les places de stationnement réservées par exemple), il recouvre en réalité une pluralité de situations, habituellement regroupées en cinq catégories principales: les déficiences motrices, visuelles, auditives, mentales et psychiques. Une très grande diversité existe néanmoins au sein même de chacune de ces catégories, rappelant à quel point il est difficile de dresser des approches catégorielles sur des questions si fortement liées à la diversité humaine elle-même.

5 Dans le cadre de cet article, nous privilégierons une approche globale du handicap, en tâchant d'en refléter la diversité par la mobilisation d'exemples variés. Pour ne citer que quelques chiffres, reprenons la proposition de Charles Gardou, fondée sur les résultats du rapport mondial sur le handicap précédemment cité : «Sur une population mondiale de 7 milliards, plus d'un milliard est en situation de handicap. Si l'on inclut les membres de leur famille - parents, fratrie, conjoints - quotidiennement impliqué, plus d'un tiers des habitants de la planète se trouve donc concerné de façon directe ou indirecte » (Gardou, 2012, p. 21). Le rapport mondial sur le handicap pointe aussi le développement que connaîtra le handicap ces prochaines années, en lien avec le vieillissement de la population globale et les changements environnementaux de différents ordres : «Le nombre des personnes handicapées est en augmentation. Cela est dû au fait que les populations vieillissent (les personnes âgées ont un risque plus élevé de handicap) et à l'augmentation mondiale des problèmes de santé chroniques associés à un handicap, comme le diabète, les maladies cardiovasculaires ou les maladies mentales» (Banque Mondiale et OMS, 2011, p. 8).

Ainsi, le handicap semble s'imposer comme une évidence démographique, mais aussi sur les plans humain et social. Et depuis une quinzaine d'années en effet, la question du handicap et de sa prise en compte (en premier lieu l'accessibilité) occupe une place grandissante dans le débat public, faisant écho à la fois à l'évolution démographique et à celle de la pensée contemporaine, et se traduisant par une inscription du handicap dans le cadre législatif et politique, tant à échelle nationale qu'internationale. En France, citons la Loi $n^{\circ}$ 2005-102 du 11 février 2005 pour l'égalité des droits et des chances, la participation et la citoyenneté des personnes handicapées. Ce texte introduit des notions fondamentales telles que celles d'accessibilité et de participation 
citoyenne. À l'échelle internationale, soulignons le caractère unificateur et fondamental de la Convention des Nations Unies relative aux droits des personnes handicapées, adoptée par l'ONU le 13 décembre 2006, et communément appelée la CRPD (de son nom anglais « Convention on the Rights of Persons with Disabilities »). Au vu du nombre important de pays signataires de cette convention (la France l'a signée le 30 mars 2007), nous pouvons la considérer comme la preuve de l'émergence et la concrétisation d'un consensus fort et réel, soudant une unité d'action et de pensée concernant la prise en compte du handicap.

7 Ce consensus conceptuel apparent est la traduction d'évolutions issues à la fois de la recherche académique (notamment des disability studies) et des mouvements identitaires menés par les personnes en situation de handicap elles-mêmes (en particulier aux États-Unis dans les années 1970), ayant amené un véritable changement de paradigme dans la façon de penser et considérer le handicap (Stiker, Ravaud et Albrecht, 2001). Le contexte international dans son ensemble encourage et traduit un changement de perspective, perceptible notamment à travers les évolutions sémantiques que révèle l'usage de certaines terminologies, en premier lieu dans les définitions juridiques et officielles (Baudot, Borelle et Revillard, 2013). Ainsi une nouvelle approche, en introduisant des tonalités plus positives (remplaçant par exemple le terme «incapacité » par celui «d'activité»), cherche à recentrer le débat sur les capacités réelles des individus concernés par le handicap. Cette approche prend davantage en compte les facteurs environnementaux ${ }^{1}:$ "L'état de fonctionnement et de handicap d'une personne est le résultat de l'interaction dynamique entre son problème de santé (maladies, troubles, lésions, traumatismes, etc.) et les facteurs contextuels. [...], les facteurs contextuels comprennent à la fois des facteurs personnels et des facteurs environnementaux» (CIF, 2001, p. 8). Dans cette perspective, c'est la société qui - par son inadaptation - est responsable du handicap : «Les barrières qui contribuent au handicap aggravent les désavantages vécus par les personnes handicapées » (Banque mondiale et OMS, 2011, p. 11). Elle a donc la responsabilité de s'adapter: la logique de mise en accessibilité se répand, y compris concernant les questions d'ERE : «Elles [les personnes en situation de handicap] ont les mêmes droits à l'éducation à l'environnement dans une perspective de développement durable et ont aussi les mêmes devoirs et la même responsabilité éco-citoyenne » (Coquillaud Auffret, 2009).

8 Ainsi, nous considérons que la pensée contemporaine du handicap se structure autour d'enjeux phares que nous allons résumer ici brièvement, avant de les mettre à l'épreuve des problématiques d'ERE. En premier lieu, soulignons encore une fois l'importance de l'accessibilité et de l'autonomie d'action, posées comme les piliers de la prise en compte sociale du handicap. La notion d'inclusion apparaît elle aussi comme fondamentale et elle est la conséquence directe de la prise de position précédente. Elle implique nécessairement un changement sociétal global, intégrant l'accessibilité et les besoins spécifiques dans tous les pans de la vie sociale. Dans cette perspective, l'accessibilité doit être pensée comme un allant de soi, et ne pas advenir dans un second temps: elle ne doit pas prendre la forme d'une adaptation a posteriori mais être au contraire intégrée dès la conception des aménagements. Ainsi, cela permettrait aux personnes en situation de handicap d'être incluses dans la société, avec leurs spécificités mais au même titre que toute autre personne, et il n'est pas de leur responsabilité individuelle de s'adapter à l'environnement, mais à la société de s'assurer de produire un environnement adapté à tous. Pour cela, la notion 
d'empowerment s'impose comme une des conditions qui précède la proposition précédente, car c'est en entendant et en prenant en considération la voix des personnes en situation de handicap que la société sera en mesure de produire les dispositifs les mieux adaptés aux besoins de chacun. Il s'agit en outre d'un enjeu fondamental de participation citoyenne.

\section{Le handicap dans les espaces naturels protégés?}

Il s'agit maintenant de comprendre de quelle façon les enjeux liés à la question du handicap et ceux associés à l'ERE vont pouvoir se concilier au regard du développement de territoires inclusifs et respectueux de la diversité des individus qui le composent. Nous verrons comment la prise en compte des personnes en situation de handicap dans les aires naturelles protégées permet d'aller dans le sens des objectifs de ces dernières, tout en respectant les injonctions à la fois éthiques et politiques de respect des normes, de mise en accessibilité et d'accueil des personnes en situation de handicap.

Dans le cas des espaces naturels, la prise en compte du handicap est ancrée dans une logique de territoire, et implique un grand nombre d'acteurs et de secteurs; elle se révèle donc un enjeu d'une ampleur considérable. En effet, se construire et se revendiquer comme territoire inclusif, accessible et respectueux des différences est un projet global, comme le rappelle Frédéric Bauer: «finalement, plus qu'un concept d'ajustement social et de compensation, le concept d'inclusion doit être entendu et utilisé comme un projet de société général et fondamental, concernant, dans sa mise en œuvre comme dans ses résultats, l'ensemble des citoyens» (Bauer, 2015, p. 73). Pour cette raison, l'analyse des territoires vécus nous semble tout à fait pertinente, car c'est par excellence le lieu de la transition sociétale que permettra le développement de démarches inclusives. L'analyse de ce type d'espace semble donc particulièrement intéressante pour comprendre ce tissage impliquant identité de territoire, enjeux de développement et traitement social du handicap.

11 Dans les faits, le croisement des thématiques de l'accessibilité et des aires naturelles est fort peu exploré. Il existe plusieurs " guides de bonnes pratiques ", articles et dossiers de type "retour d'expérience $»^{2}$, surtout dans la littérature grise, qui renseignent avec plus ou moins de précision les actions initiées en direction des personnes en situation de handicap, mais nous faisons le constat du peu de retours réflexifs sur ces initiatives, qui restent au demeurant anecdotiques, ponctuelles et partielles, comme le rappelle ici cet acteur de terrain engagé en faveur de l'accessibilité dans les espaces d'ERE : «Il existe peu d'outils d'éducation à l'environnement spécifiques aux personnes déficientes. Soit l'animateur utilise les outils traditionnels de découverte sensorielle, soit il fait confiance à son expérience, soit il crée lui-même un outil.» (Interview de Paul Gailly, responsable du service éducatif de l'association environnementale Natagora, dans Dubois, 2013, p. 9).

À cet effet, on constate la nécessité de mener des recherches académiques interdisciplinaires, croisant la géographie sociale, le champ de l'ERE, la sociologie du handicap et la muséologie pour aborder la question du handicap dans les aires naturelles protégées, faisant apparaître à la fois la complexité des enjeux qui y ont associés, les solutions envisagées et la façon dont cela révèle les représentations contemporaines et le traitement social du handicap. En conditionnant l'expérience de visite des personnes en situation de handicap (par l'instauration de parcours ou de 
dispositifs spécifiques de médiation culturelle, par exemple), les espaces comme les musées, les centres d'interprétations ou les parcs naturels contribuent à la construction sociale et identitaire du handicap.

\section{Protéger et adapter la nature : des objectifs inconciliables ?}

Dans un premier temps, prenons en considération un des enjeux phares de la création d'espaces naturels protégés, qui semble s'imposer comme une évidence : celui de la protection de la nature. Nous acceptons la proposition de Patrick McKeever, secrétaire du Programme international de géosciences de l'UNESCO, mentionnée dans l'article de Catalina Gonzalez-Tejada et coll. (2017, p. 7) : « le Géoparc est un concept holistique qui combine trois objectifs principaux : la protection du patrimoine géologique, l'éducation et le développement local durable». Le premier enjeu, celui de la protection de la nature, est notamment soutenu par la patrimonialisation de cette dernière mais aussi par le soutien au développement de la recherche par le biais de l'institutionnalisation des aires naturelles (la création de programmes de protection et labellisation à échelle européenne peut par exemple aller dans ce sens).

Ne pas « dénaturer » le territoire et ses paysages s'impose donc comme une mission de premier ordre. Nous sommes là face à une des contradictions fondamentales de ce type de démarche: comment protéger la nature de l'intervention humaine tout en produisant des aménagements en vue de la valoriser? Les aménagements dans le cas des visiteurs en situation de handicap sont indispensables, mais comment peuvent-ils s'intégrer au mieux au sein de cette ambition ? Car l'accessibilité est fondamentale : elle est perçue, affirmée et revendiquée comme le garant d'une égalité citoyenne (Nations Unies, 2006). En effet, la société porte la responsabilité de s'adapter pour permettre à chacun d'accéder de façon égalitaire à tous les champs de la vie sociale. Rappelons donc l'importance de l'accessibilité à l'expérience de sortie et de visite des personnes en situation de handicap. Si elle n'est pas le seul facteur de décision et de satisfaction dans la visite d'un musée, l'accessibilité reste un critère essentiel (Lebat, 2013). La crainte de se trouver dans un lieu difficile d'accès ou dans lequel les prestations ne sont pas accessibles, peut freiner considérablement l'élaboration d'un projet de visite, voire amener la personne à y renoncer. Ainsi, le manque d'accessibilité des sites naturels et touristiques entraîne une restriction de la liberté de choix : « Les critères de choix de la destination sont centrés sur le facteur accessibilité avec une attente forte pour un personnel d'accueil formé » (Blaho-Ponce, 2013, p. 108).

Pour faire entrer cet impératif au sein des espaces naturels, le code mondial d'éthique du tourisme en rappelle l'importance: "la possibilité d'accéder, directement et personnellement, à la découverte des richesses de la planète constitue un droit également ouvert à tous les habitants du monde. La participation toujours plus étendue au tourisme local, national ou international (...) ne doit pas se voir opposer d'obstacles» (Organisation Mondiale du Tourisme, 1999 cité par Blaho-Ponce, 2013, p. 104). Le récent rapport du Sénat sur l'accès à la culture pour les personnes en situation de handicap rappelle lui aussi cette exigence car il pose l'accessibilité et l'égalité d'accès aux lieux culturels pour les personnes en situation de handicap comme des « exigences démocratiques » (Duranton et Gonthier-Maurin, 2017). Néanmoins, les espaces voués à la protection ou la valorisation de l'environnement rencontrent des difficultés qui leur sont propres, car la mise en accessibilité nécessite des 
aménagements spécifiques, alors même qu'une de leurs ambitions principales est précisément de limiter toute intervention humaine invasive.

L'idée de préservation d'une nature la plus vierge possible est au cœur de l'idéologie qui sous-tend la création d'espaces naturels protégés. Il y a donc là une tension certaine entre la volonté de préservation d'une nature "vraie » et l'accueil de divers publics dans le cadre de programmes d'éducation relative à l'environnement. Les acteurs engagés tentent alors de privilégier des aménagements respectueux de l'environnement, ayant un moindre impact sur ce dernier. Cela se traduit par une attention portée aux matériaux utilisés par exemple, privilégiant des matériaux organiques comme le bois :

Aménager un site naturel pour le rendre accessible implique la double contrainte de domestiquer la nature tout en la respectant. [...] Platelage en bois locaux, tunnels végétaux, pontons, utilisation du braille sur les panneaux extérieurs, sentiers sur pilotis, chemins en enrobé végétal... tout doit se fondre dans le paysage pour un impact environnemental réduit. (Nick, 2011, p 13).

17 Il s'agit de préférer des éléments et matériaux durables et profitables à tous, afin de garantir une accessibilité maximale autant qu'un impact maîtrisé sur la nature. Bien entendu, cet impératif de protection de la nature amène dans certains cas les acteurs impliqués à faire le choix de limiter l'accès et n'aménager qu'une partie restreinte du site pour y accueillir les visiteurs. C'est le cas de la majorité des parcs naturels, même lorsqu'ils sont dits adaptés : seule une partie des sentiers (souvent un seul sentier) est aménagée, afin de limiter le développement d'aménagements accessibles.

Ainsi certains aménagements prennent en considération l'accessibilité tout en tentant de garantir un respect et une protection optimale de la nature. Ils doivent concilier ce premier impératif avec celui de la valorisation et de la diffusion du patrimoine naturel : il s'agit là d'un deuxième enjeu sur lequel nous insisterons. Comment protéger la nature sans la mettre "sous cloche", mais au contraire en y proposant un accès raisonné et permettant l'éducation et la sensibilisation de ses visiteurs?

\section{Assurer l'accessibilité des initiatives de sensibilisation et d'éducation : différentes logiques de traitement social du handicap}

Les espaces comme les géoparcs et les activités qui les accompagnent sont considérées comme des moyens efficaces de sensibiliser et d'éduquer aux principales problématiques environnementales, comme le changement climatique (Zouros, 2012). Cet enjeu est au cœur de l'éducation relative à l'environnement contemporaine et prend notamment forme dans des espaces tels que des centres d'interprétation ou les musées de sciences. On se rapproche alors des champs de la muséologie (et notamment muséologie de plein air) et de la médiation scientifique, à travers lesquels on observe différentes pistes pour la prise en compte du handicap, en particulier depuis une quinzaine d'années en France (sous l'influence de la loi de 2005 précédemment citée) et dans le monde. En témoigne la multiplication des guides de bonnes pratiques, retours d'expérience ou articles scientifiques publiés notamment dans la littérature muséologique. Nous allons présenter plusieurs exemples de dispositifs en place dans les musées et les espaces naturels, et à travers eux se révéleront certaines conceptions et logiques sociales de traitement du handicap. Car si l'accessibilité est au centre de toute 
démarche d'accueil des personnes en situation de handicap, elle peut répondre de logiques distinctes.

Certains dispositifs relèvent ou tâchent de relever d'une muséologie ou muséographie inclusive, faisant l'usage des principes de la conception universelle (ou universal design). Ces dispositifs visent alors une autonomie du visiteur, tout en étant naturellement, facilement et intuitivement utilisables par tous, quels que soient l'origine, la déficience ou l'âge de la personne. Un des points fondamentaux de cette approche est de tenir compte de la mixité des publics, en respectant les spécificités de chacun. Cet objectif complexe - celui de l'inclusion - est vécu à la fois comme une difficulté et comme un défi au sein des institutions muséales. Malgré la complexité de la tâche, certaines institutions ont développé des projets novateurs et créatifs, tâchant de tendre vers cette universalité. Dans les aires protégées, citons la mise en place de chemins universels (ou sentiers universels) : ils sont conçus pour s'adapter à tous, dans le respect et la reconnaissance des différences et des capacités propres à chacun. Ils présentent donc une accessibilité physique optimale (notamment pour assurer un accès aux fauteuils roulant) et développent une médiation accessible à la fois aux personnes voyantes (textes en noir), déficientes visuelles (braille et gros caractères), et avec différents niveaux de lecture pour s'adapter au niveau cognitif et à la maîtrise de la langue de chacun (adultes, enfants, personnes déficientes intellectuelles, étrangers ne maitrisant pas la langue, etc.). La visée de ce type de dispositifs est d'activer les capacités propres à chaque individu en lui permettant de se saisir de son expérience par le biais de ses propres modalités : ils favorisent une liberté d'appropriation pour chaque visiteur.

21 Ces démarches encouragent l'expérience autonome de chacun, sans avoir nécessairement besoin de l'aide d'une personne accompagnatrice ; l'accessibilité ainsi pensée a pour objectif de ne pas placer le handicap au centre de l'expérience (mais sans nier la déficience, ce qui relèverait bien entendu d'une démarche adaptative) et de permettre à l'individu en situation de handicap d'accéder d'une manière égalitaire aux différents espaces et aux différentes activités proposées dans ces lieux. Cela répond tout à fait à la logique de la loi de 2005 et s'ancre de façon évidente dans le paradigme social du handicap porté par les chercheurs notamment anglo-saxons du champ des disability studies. Notons que l'injonction à l'autonomie ne prend pas nécessairement la forme de dispositifs inclusifs; certaines approches catégorielles sont conçues autour de cette idée d'autonomie, comme par exemple les bandes podotactiles ${ }^{3}$ et les plans en relief (ou maquettes) dans les musées. Ce type de dispositifs explicitement dédiés aux visiteurs déficients se fondent sur l'idée qu'une autonomie totale est possible. En leur apportant spécifiquement les éléments nécessaires à l'orientation et aux déplacements autonomes, ces dispositifs sont censés permettre des visites indépendantes, sans assistance extérieure. Pour les visiteurs sourds, l'équipement des salles en boucles magnétiques permet de suivre une visite de façon absolument similaire aux visiteurs entendants; la présence de visio-guides ou d'interprétation en langue des signes s'ancre dans cette même logique. Ainsi, un dispositif peut garantir l'accessibilité d'une offre en assurant un accès autonome et complet au contenu, mais sans permettre la mixité des publics.

22 Ces démarches, qu'elles soient inclusives, catégorielles ou mixtes, sont centrées autour de la notion d'autonomie, qui semble s'imposer comme une valeur fondamentale et fondatrice de la notion même d'accessibilité. Toutefois, en s'interrogeant sur le sens 
que cela prend pour les personnes en situation de handicap elles-mêmes, et les apports en termes de satisfaction et d'expérience de visite, nous pourrons remettre en question sa centralité et sa capacité à être le (seul) garant d'une accessibilité « réussie ». En effet, "le principe d'autonomie semble être devenu aujourd'hui l'alpha et l'oméga de l'éthique médicale » (Svandra, 2007, p. 75), et dans les espaces muséaux ou d'accueil cela se vérifie aussi. Mais du point de vue des visiteurs eux-mêmes, l'autonomie absolue n'est presque jamais revendiquée (Lebat, 2018), car la sortie est fondée sur des attentes et des pratiques similaires aux autres visiteurs, en partie fondée sur une envie de partage et de sociabilité. Ainsi, le rôle de l'accompagnateur - que les dispositifs visant une autonomie complète tendent à faire disparaître ou en tout cas à rendre optionnel est central dans la visite, et ceci pas uniquement pour l'aide qu'il apporte, mais aussi pour le partage et la convivialité qu'il permet.

La question de l'autonomie est donc au cœur de l'expérience de sortie, et c'est aussi autour d'elle que se sont développées d'autres conceptions du traitement du handicap qui trouvent des échos dans les espaces d'ERE par la mise en place de dispositifs adaptés. Certains dispositifs sont en effet développés en intégrant la question de l'autonomie mais en ne la posant pas comme un impératif absolu: ils acceptent la nécessité de l'accompagnement de la personne dans sa découverte de l'espace. Si l'on considère l'autonomie absolue comme un leurre ou comme une "fausse piste", ces démarches s'ancrent dans une logique sociétale basée sur la solidarité et des systèmes d'interdépendances positives.

Cette logique est notamment développée dans les travaux portant sur la notion de care, qui avancent une éthique nettement éloignée de l'approche sociale des disability studies. En effet, l'éthique du care postule la dépendance comme un élément constitutif de l'être humain, et un des fondements de la vie sociale. En ce sens, l'injonction à l'autonomie est relativisée: il s'agit au contraire de développer les relations interindividuelles fondées sur l'entraide, qui est vidée de la dimension avilissante que lui attribue la pensée sociale contemporaine. L'injonction à l'autonomie apparait donc en réalité aussi violente que l'assignation à une relation de dépendance. « Il faut se montrer aussi forts que les forts et les valides» (Stiker, 2009, p. 53): cette obligation sociale est une pression normative qui s'impose aux personnes en situation de handicap, et contre laquelle se positionnent les théories du care. Car comme le précise Myriam Winance, « une personne autonome n'est pas une personne qui décide et agit seule, mais dont le pouvoir décisionnel et les capacités d'action sont soutenus par de multiples relations (sociales, techniques, institutionnelles, symboliques...) » (Winance, 2007, p. 84).

Dans les démarches d'accessibilité développées dans les espaces naturels ou muséographiques d'ERE, cela peut se traduire par le recours à l'accompagnement humain plus qu'au développement de dispositifs visant l'autonomie. Une fois la dépendance à autrui débarrassée de son sens négatif, mais au contraire replacée dans une norme constitutive de l'humain, alors les démarches peuvent être fondées sur l'aide humaine. C'est par exemple le cas à la Cité des Sciences et de l'Industrie, qui propose à ses visiteurs en situation de handicap un accompagnement : sur demande, les visiteurs aveugles peuvent être accompagnés pendant toute leur visite par un médiateur, qui les guident même dans leurs trajets - aller et retour - du métro jusqu'à l'entrée du musée. Citons aussi l'exemple des joëlettes, outil auquel ont recours nombre de sites naturels dans leur démarche de mise en accessibilité : ce fauteuil de randonnée pour personnes à mobilité réduite permet à l'individu de parcourir des sentiers de 
randonnées qui sont inaccessibles aux fauteuils roulants classiques, mais il nécessite l'aide de tiers. Le site d'escalade adapté pour les personnes déficientes visuelles dans le parc du Morvan (dans le cadre du projet « Morvan pour tous » (Guide du Morvan pour tous, édité par le Parc naturel régional du Morvan) est également fondé sur cet équilibre entre autonomie d'action (par un guidage sonore apporté aux personnes déficientes visuelles souhaitant escalader) et recours à l'aide d'un tiers (qui accompagne et encadre l'activité). Il s'agit aussi de replacer la notion de sociabilité au cœur de l'expérience de sortie, postulant que cette dernière est plus forte que celle d'autonomie, et que si elle entraine une collaboration entre la personne en situation de handicap et son accompagnateur, cela n'est en rien incompatible avec la reconnaissance du handicap et le respect de la personne. L'éthique du care ne place pas l'autonomie au cœur des revendications, et plus encore : elle nie son utilité sociale.

Cependant, il s'agit là d'une approche un peu radicale et qui anticipe un changement sociétal majeur, et des mutations profondes dans les considérations des rapports humains. De plus, cela peut aussi être perçu comme le reflet de la persistance de logiques discriminatoires et ségrégatives, par le manque concret d'accessibilité, par des approches catégorielles (étiquetage) ou la limitation des modalités d'appropriation du monde (par la restriction de la mobilité et des approches sensorielles, ce dernier point concernant essentiellement les visiteurs déficients sensoriels). L'expérience de visite est profondément modifiée par certains éléments et choix opérés, et imposés le plus souvent sans consultation et implication des personnes en situation de handicap ellesmêmes.

\section{Accessibilité et développement territorial}

27 Le développement économique local (et notamment par le géo- ou éco-tourisme) apparaît très clairement comme une des motivations majeures à la création d'espaces et de dispositifs favorisant l'accueil de personnes en situation de handicap. En effet, la mise en place du label géoparc est né $\mathrm{du}$ «désir de stimuler le développement économique dans les régions rurales, principalement par le biais de la promotion du tourisme » (Gonzalez-Tejada et coll, 2017, p. 2, traduction libre). En 2012 déjà, Nicolas Zouros, président du réseau mondial des géoparcs, soulignait leur impact sur le développement économique local: «ce qui est encore plus important pour l'emploi dans la région, c'est le nombre d'autres emplois créés dans les entreprises touristiques, les petits hôtels, les pensions, les restaurants et d'autres activités liées à l'augmentation du flux touristique dans la région du Géoparc» (Zouros, 2012, p. 40). Dans une telle perspective, l'accueil du handicap doit trouver une place.

L'accessibilité relève d'une prise en compte globale du territoire, car elle repose sur le concept de chaîne d'accessibilité. Elle doit en effet s'intégrer dans chaque étape du parcours de visite ou de sortie d'un individu, et cela dans un sens large, commençant bien avant l'arrivée sur le lieu (comme la recherche d'information ou l'identification de trajets pour se rendre au lieu), se développant sur le lieu de l'expérience recherchée (accès aux espaces et aux services) et se poursuivant à la suite de cette dernière (entre autres, le retour au domicile et la possibilité de prolonger par l'accès à des ressources complémentaires.).

Or la communication par exemple, qui constitue un préalable indispensable, est très souvent non adaptée et devient par conséquent un frein capital : «Un des obstacles 
majeurs à l'accès des personnes handicapées aux espaces naturels, est le manque d'information fiable sur l'accessibilité des sites » (Nick, 2011, p. 7). Nous avons d'ailleurs pu observer au cours de nos recherches, le manque de lisibilité des sites Internet des aires naturelles protégées et la difficulté à obtenir via ces derniers une information complète, fiable et d'actualité concernant l'accessibilité réelle du lieu. Il en est de même pour les déplacements: "Il est impératif de favoriser une chaîne cohérente de déplacements quelle que soit la déficience de la personne handicapée » (Blaho-Ponce, 2013, p. 109). Isabelle Vandenbosch, actrice de l'ERE à destination des personnes en situation de handicap en Belgique, souligne la nécessité de préparer les trajets et déplacements : «Il est important de repérer les lieux. Un obstacle imprévu - on arrive avec une personne en chaise roulante au pied d'un escalier et on doit faire demi-tour peut générer une frustration. Certaines personnes peuvent alors se refermer et se mettre en situation mentale de handicap. Elles vont subir la suite... » (Interview de Isabelle Vandenbosch, présidente de SEL Bleu, cité dans Dubois, 2011, p. 8). Ainsi, la mise en accessibilité du lieu en lui-même, si elle est capitale, est en soi insuffisante :

L'accessibilité culturelle implique plusieurs types d'opérateurs et d'actions. Cet ensemble constitue la chaîne de l'accessibilité culturelle. Si l'un des maillons de cette chaîne vient à manquer, la personne en situation de handicap sera privée de son accès à la pratique culturelle. Cette chaîne de l'accessibilité culturelle mobilise un ensemble d'acteurs: culturels, sociaux, médico-sociaux, associatifs, ... (Cémaforre, 2008, p. 2)

Tous les acteurs d'un territoire sont amenés travailler ensemble, en réseau, afin de favoriser une continuité dans l'offre adaptée. Cette démarche peut créer une cohérence de discours, amener les acteurs à se rencontrer et à harmoniser leurs prestations. Cette cohérence de discours et de posture peut notamment se concrétiser par la création et l'obtention de labels, dont le principal en France à ce jour est le label «Tourisme et handicap ». C'est le point de vue développé par Frédéric Reichhart et Aggée Lomo : les labels peuvent être un moyen de diffusion et d'harmonisation des "bonnes pratiques » et inciter les sites à s'investir dans les démarches de mise en accessibilité. Ces auteurs font d'ailleurs le lien entre l'absence d'uniformisation au niveau des labels et le manque de cohérence et de lisibilité de l'offre. Le développement des labels pourrait apporter une réponse à des offres qui apparaissent jusqu'alors comme disparates, désordonnées, et donc peu compréhensibles, même pour les personnes à qui elles sont adressées : « À défaut d'un label européen, il existe sur le terrain une multitude de labels, chartes et pictogrammes nationaux. Cette situation explique le patchwork des labels et pictogrammes qui aboutit à une carte de l'accessibilité relativement morcelée et illisible » (Reichhart et Lomo, 2013, p. 83). Cependant, les labels ont pour l'instant une portée trop réduite pour jouer véritablement ce rôle d'incitation à la mise en accessibilité.

31 Ainsi, en ayant un impact sur l'ensemble des dispositifs en place sur le territoire et impliquant chacun de ses acteurs, l'accessibilité prend une place capitale au sein du développement local et l'aménagement du territoire. Elle est au cœur de ce développement territorial, et notamment économique, qui est aussi un des enjeux fondateurs des projets d'institutionnalisation des espaces naturels protégés. Ce développement économique local est souvent fondé sur le développement (éco)touristique, et la prise en compte des personnes en situation de handicap y est un enjeu de taille: nous l'avons vu en introduction de cet article, le handicap s'impose comme une évidence sur le plan humain, social, mais aussi touristique et financier, si 
l'on prend en considération les impacts économiques de l'accroissement démographique de cette population: "l'offre touristique à l'égard des personnes en situation de handicap est un marché porteur. D'ailleurs l'étude Touch Ross estimait en 1993 que 36 millions d'européens en situation de handicap étaient financièrement solvables et aptes à voyager » (Amiaud, 2012, p. 53).

L'accessibilité incluse dans tous les pans de l'activité d'un territoire, peut être vécue comme une contrainte extrêmement forte mais elle apparait finalement comme une force au profit du développement local. Elle induit en effet de nécessaires coopérations entre les différents acteurs, fondant une cohésion d'action et de discours: tout cela contribue à une redynamisation du territoire, attirant un public (et par extension une clientèle nouvelle), motivant la communication et incitant à la mise en place de partenariats. Ainsi, l'impératif de mise en accessibilité peut amener à renforcer un territoire et aboutir à une appropriation plus forte de l'identité de ce dernier par les acteurs impliqués.

\section{Identité de territoire et empowerment}

33 En effet, l'implication de la population locale et le développement d'un sentiment d'appartenance à un territoire est un objectif qui sous-tend la mise en place de ce type d'espace. Les habitants sont amenés à devenir des ambassadeurs de leur territoire, ce que la déclaration d'Arouca, adopté à l'issue du Congrès International de Géotourisme de 2011, exprime clairement et ce, dès ses premières lignes : » le géotourisme doit être défini comme un tourisme qui soutient et améliore l'identité d'un territoire » (2011, article 1). Plus loin, on peut lire ceci : « nous recommandons que la population locale et les visiteurs soient impliqués d'une manière efficace et ne se limitent pas à un simple rôle de spectateurs, en contribuant à construire ainsi une identité locale, promotionnant les valeurs authentiques et uniques du territoire " (article 6). L'identité de territoire constitue donc le dernier enjeu sur lequel nous insisterons, en montrant comment la prise en compte des personnes en situation de handicap peut s'y intégrer, voire même aider à son développement. Pour cela, deux points nous semblent centraux : l'accessibilité physique bien sûr, la possibilité d'accéder aux espaces, mais aussi l'implication, l'empowerment, induisant une reconnaissance pouvant aisément créer les conditions de l'avènement d'un sentiment d'appartenance.

Cela s'active par l'implication des différents acteurs à des niveaux différents on l'a vu, mais aussi et évidemment, par l'implication des personnes en situation de handicap elles-mêmes. La notion d'empowerment constitue un des fondements de la pensée du handicap telle que développée et diffusée par les mouvements identitaires anglosaxons et repris notamment par le champ académique des disability studies. Cela peut s'illustrer par exemple, par la mise en place de collaborations avec des associations locales, qui prennent souvent la forme de séances de test des dispositifs (comme les stations tactiles). Citons également cette initiative menée dans la Réserve Naturelle de Séné. Une collaboration a été instaurée avec un foyer d'hébergement de personnes déficientes intellectuelles, dans le but de concevoir ensemble des livrets rédigés avec la méthode dite "facile à lire et à comprendre ${ }^{4}$ destinés à l'accompagnement de la découverte de cette aire protégée :

Cette année [2017), la Réserve Naturelle de Séné a débuté une collaboration avec le foyer d'hébergement "Les Bruyères", situé à Plumelec; toutes les 3 ou 4 semaines, un petit groupe de résidents du foyer est accueilli à la Réserve Naturelle pendant 
$2 \mathrm{~h} 30$ à $3 \mathrm{~h}$ de temps partagé entre balade sur le terrain et discussion en salle. Pour débuter, le petit groupe de travail s'est fixé comme but d'élaborer un livret de visite sur la Réserve. Rédigé selon la méthode "Facile à lire et à comprendre" (méthode européenne permettant une accessibilité des informations aux personnes déficientes intellectuelles), il sera destiné aux personnes présentant des difficultés à lire, et pourra aussi servir aux jeunes enfants en apprentissage de la lecture ou aux personnes étrangères. ${ }^{5}$

Cette collaboration permet d'une part, la création d'un outil utilisable avec des publics multiples, mais permet aussi de créer un lien fort entre ce groupe et son territoire, par l'implication réelle des participants. La création d'un lien avec le territoire vécu est un enjeu fondamental car, comme le soulignent Yves Girault et Angela Barthes (2016, paragr. 22), «le territoire est un "espace informé" qui construit une compréhension du monde et une identité ». Y impliquer les habitants, les acteurs, les individus de manière tout à fait personnelle est essentiel pour les amener à s'approprier un environnement. Car si l'accessibilité est fondée sur la volonté de garantir la capacité à se mouvoir et à accéder physiquement aux espaces, elle doit aussi accompagner la capacité d'un individu à se représenter et s'approprier l'environnement. Cela peut également être encouragé par la mise en place d'outils d'appréhension de l'espace, tels que les maquettes ou les plans tactiles en relief pour visiteurs déficients visuels.

\section{Conclusion}

Accéder de manière libre et aisée à son environnement, c'est donc obtenir les clés pour se l'approprier et agir sur lui (tant pour le modifier que pour le préserver): «Une première étape d'éducation relative à l'environnement consiste à explorer et redécouvrir son propre milieu de vie, explorer "l'ici et maintenant" des réalités quotidiennes avec un regard neuf, appréciatif et critique à la fois " (Sauvé, 2002, p. 2). En exclure un citoyen du fait de son handicap et le garder à distance relève d'un processus de discrimination : cela constitue une forme d'exclusion de la communauté (Blanc, 2006). Les enjeux de l'accès aux espaces naturels protégés sont donc l'expression de l'absolue nécessité de la prise en considération des personnes en situation de handicap.

L'attention portée au handicap et notamment aux impératifs d'accessibilité est souvent vécue comme une contrainte. Or nous avons montré qu'elle n'est en rien incompatible avec les enjeux propres aux territoires protégés et aux acteurs qui y agissent et y évoluent. Les initiatives d'accessibilité sont inscrites dans un projet sociétal global d'accueil de la différence, que les multiples actions et postures en place contribuent tout à la fois à construire, à renseigner et à diffuser. 


\section{BIBLIOGRAPHIE}

Amiaud, D. (2012). Tourisme et handicap : recherche sur les conditions d'accessibilité aux aménités du littoral. Thèse de doctorat. Université de La Rochelle.

Banque Mondiale et Organisation Mondiale de la Santé. (2011). Rapport Mondial sur le Handicap. Genève : Éditions de l'OMS.

Baudot, P-Y., Borelle, C. et Revillard, A. (2013). Politiques du handicap. Terrains \& travaux, 23(2), 515.

Bauer, F. (2015). Inclusion et planification : vers un territoire inclusif. Vie sociale, 11(3), 71.

Blaho-Ponce, C. (2013). La chaîne d'accessibilité, pivot de l'accès au Tourisme Handicap. Téoros. Revue de recherche en tourisme, 32(2), 104-115.

Blanc, A. (2006). Le handicap ou le désordre des apparences, Paris : A. Colin.

Bergerie Nationale et ARENE Ile-de-France (2009). Éducation à l'environnement et handicap. Réaliser son diagnostic pour réussir l'accueil en ferme pédagogique et les autres structures. Rambouillet : Les cahiers techniques de la Bergerie Nationale.

Cémaforre (2008). Fiche pratique « La chaîne de l'accessibilité », Pôle européen de l'accessibilité culturelle / European center for cultural accessibility. Paris : Éditions Cémaforre.

Coquillaud-Auffrey, M-S. (2009). L'éducation à l'environnement pour les personnes en situation de handicap. Pour, 202-203, 16-20.

Dubois, C. (2011). Éducation à l'environnement et handicaps. Symbioses, le magazine de l'Éducation Relative à l'Environnement, 89, 8-9.

Duranton N. et Gonthier-Maurin, B. (2017). Rapport d'information fait au nom de la commission de la culture, de l'éducation et de la communication. Groupe de travail « Culture et handicap ». Rapport $N^{\circ}$ 648. Paris : SÉNAT.

Gardou, C. (2012). La société inclusive, parlons-en! il n'y a pas de vie minuscule, Toulouse : Érès. Forum des gestionnaires. (2009). L'accessibilité de tous les publics : pour une nature ouverte à tous. Actes du 15è forum des gestionnaires, Lille.

Girault, Y. et Barthes, A. (2016). Postures épistémologiques et cadres théoriques des principaux courants de l'éducation aux territoires, Éducation relative à l'environnement [En ligne], Volume 13 2

Gonzalez-Tejada, C., Du, Y., Read, M. [et al.]. (2017). From nature conservation to geotourism development : Examining ambivalent attitudes towards UNESCO directives with the global geopark network, International journal of Geoheritage. 5 / ISS 2, 1-20.

Lebat, C. (2013). Déficience visuelle et pratiques culturelles : résultats de deux enquêtes qualitatives. Rapport d'enquête. Paris : RECA, Ministère de la Culture et de la Communication. En ligne : www.culturecommunication.gouv.fr/content/download/114522/1306769/version/1/file/ enquete-RECA-DV-synthese.pdf.

Lebat, C. (2018). L'accueil des personnes en situation de handicap sensoriel dans les musées : réalités d'accueil, expérience de visite et trajectoires identitaires. Université Sorbonne Nouvelle Paris 3. 
Nick, S. (2011). Guide pour l'accessibilité dans les espaces naturels. Association C4D, Communication pour le développement durable. En ligne : www.c4dev.org.

Organisation des Nations Unies. (2006). Convention relative aux droits des personnes handicapées et Protocole facultatif. Consultable en ligne : http://www.un.org/french/disabilities/default.asp? id $=1413$

Organisation Mondiale de la Santé. (2001). Classification internationale du fonctionnement, $d u$ handicap et de la santé : CIF, Genève : éditions Weltgesundheitsorganisation.

Proceedings of the 11th European Geoparks Conference : 19 - 21 September 2012, Arouca Geopark, Portugal ; smart, inclusive, sustainable growth (2012). Arouca : European Geoparks Conference et Artur Abreu Sá.

Reichhart, F. et Lomo A. (2013). Quel tourisme pour les personnes handicapées ? Enjeux et pratiques du tourisme adapté. Téoros. Revue de recherche en tourisme. 32(2), 81-85.

Sauvé, L. (2002). L'éducation relative à l'environnement : possibilités et contraintes. Connexion, La revue d'éducation scientifique, technologique et environnementale de l'UNESCO, 27 / 1/2,1-4.

Stiker, H-J., Ravaud, J-F., et Albrecht, G. (2001). L'émergence des disability studies : état des lieux et perspectives. Sciences sociales et santé, 19(4), 43-73.

Stiker, H-J. (2009). Les métamorphoses du handicap de 1970 à nos jours : soi-même, avec les autres, Grenoble : Presses universitaires de Grenoble.

Svandra, P. (2007). L'autonomie comme expression des « capabilités ». Éthique \& Santé, $4(2), 74-77$.

Winance, M. (2007). Dépendance versus autonomie... De la signification et de l'imprégnation de ces notions dans les pratiques médicosociales : Commentaire. Sciences sociales et santé, 25(4), 83

\section{NOTES}

1. Selon la CIF (2001), «les facteurs environnementaux désignent l'environnement physique, social et attitudinal dans lequel les gens vivent et mènent leur vie ». Classification internationale $d u$ fonctionnement, du handicap et de la santé.:CIF, Éé. Weltgesundheitsorganisation, Genève, 2001, 304 p., p. 15

2. Citons par exemple : Nick, 2011 ; Forum des gestionnaires, 2009 ; Bergerie Nationale et ARENE Ile-de-France, 2009; ou encore le site internet des Parcs Naturels Régionaux français, qui propose un recueil de quelques exemples de «bonnes pratiques» sous forme de fiches détaillant les actions déjà initiées dans des parcs naturels pour accueillir les personnes en situation de handicap : http://www.parcs-naturels-regionaux.fr/centre-de-ressources/document/fichesexperiences-accessibilite-dans-les-parcs-naturels-regionaux.

3. Les bandes podotactiles - ou bandes d'appel de vigilance - sont des dispositifs à destination des personnes déficientes visuelles. En relief, elles permettent à ces derniers de repérer un trajet grâce à une canne.

4. La méthode «facile à lire et à comprendre " (FALC) est un ensemble de normes d'écriture et de rédaction élaborées en vue de permettre une compréhension optimale aux personnes déficientes intellectuelles.

5. Projet en ligne : http://reserves-naturelles.org/actualites/rnn-des-marais-de-senepartenariat-adapei-2017-2018 Consulté le 18 février 2020. 


\section{RÉSUMÉS}

Cet article explore la prise en compte de la situation de handicap au sein des espaces d'éducation relative à l'environnement. À travers des exemples concrets d'actions mises en place dans différents types d'espaces, il traite des enjeux relatifs à l'accueil des publics concernés. Ce texte mettra en évidence la façon dont les acteurs de l'éducation relative à l'environnement peuvent prendre en compte le développement inclusif des territoires, assurant une pleine participation des habitants dans toute leur diversité, tout en s'appuyant sur un modèle économique dynamique et en maintenant une exigence accrue de protection de la nature et de pédagogie environnementale.

This article aims to explore the consideration of disability within environmental education spaces. Through concrete examples of actions implemented in different types of spaces, it aims to reveal the issues related to the reception of visitors with disability. This text will highlight the ways in which environmental education actors can move towards inclusive territorial development perspectives, ensuring full participation of inhabitants in all their diversity, but relying on a dynamic business model and maintaining an increased demand for nature protection and environmental education.

\section{INDEX}

Keywords : Access, disability, environmental education, inclusion, participation

Mots-clés : accessibilité, éducation relative à l'environnement, handicap, inclusion, participation

\section{AUTEUR \\ CINDY LEBAT}

Cindy Lebat est docteure en Sciences de l'Information et de la Communication (Université Sorbonne Nouvelle Paris 3, CERLIS). Ses thèmes de recherche s'orientent autour de l'accueil des personnes en situation de handicap sensoriels dans les musées, et de leur expérience de visite. Ses travaux portent particulièrement sur les modes d'appropriation du dispositif muséal par ces visiteurs, et sur l'impact de la visite sur l'expérience vécue du handicap. 